\title{
Application of a methicillin-resistant Staphylococcus aureus risk score for community-onset pneumonia patients and outcomes with initial treatment
}

\author{
Besu F. Teshome $e^{1,2,3}$, Grace C. Lee ${ }^{2,3}$, Kelly R. Reveles ${ }^{2,3}$, Russell T. Attridge ${ }^{4,5}$, Jim Koeller ${ }^{2,3}$, Chen-pin Wang ${ }^{6}$,
} Eric M. Mortensen ${ }^{7,8}$ and Christopher R. Frei ${ }^{2,3^{*}}$

\begin{abstract}
Background: Community-onset (CO) methicillin-resistant Staphylococcus aureus (MRSA) pneumonia is an evolving problem, and there is a great need for a reliable method to assess MRSA risk at hospital admission. A new MRSA prediction score classifies CO-pneumonia patients into low, medium, and high-risk groups based on objective criteria available at baseline. Our objective was to assess the effect of initial MRSA therapy on mortality in these three risk groups.

Methods: We conducted a retrospective cohort study using data from the Veterans Health Administration (VHA). Patients were included if they were hospitalized with pneumonia and received antibiotics within the first $48 \mathrm{~h}$ of admission. They were stratified into MRSA therapy and no MRSA therapy treatment arms based on antibiotics received in the first $48 \mathrm{~h}$. Multivariable logistic regression was used to adjust for potential confounders.

Results: A total of 80,330 patients met inclusion criteria, of which $36 \%$ received MRSA therapy and $64 \%$ did not receive MRSA therapy. The majority of patients were classified as either low (51\%) or medium (47\%) risk, with only $2 \%$ classified as high-risk. Multivariable logistic regression analysis demonstrated that initial MRSA therapy was associated with a lower 30-day mortality in the high-risk group (adjusted odds ratio 0.57; $95 \%$ confidence interval 0.42-0.77). Initial MRSA therapy was not beneficial in the low or medium-risk groups.
\end{abstract}

Conclusions: This study demonstrated improved survival with initial MRSA therapy in high-risk CO-pneumonia patients. The MRSA risk score might help spare MRSA therapy for only those patients who are likely to benefit.

\section{Background}

Pneumonia is a major cause of mortality in the United States, with a reported 49,597 deaths in 2010 [1]. Community-onset (CO) pneumonia is defined as pneumonia that occurs in the community and up to $48 \mathrm{~h}$ into hospital admission. It encompasses both community-acquired pneumonia (CAP) and healthcare-associated pneumonia (HCAP). Importantly, HCAP patients are at increased risk of methicillin-resistant Staphylococcus aureus (MRSA)

\footnotetext{
* Correspondence: freic@uthscsa.edu

${ }^{2}$ Pharmacotherapy Division, College of Pharmacy, The University of Texas at Austin, Austin, TX, USA

${ }^{3}$ Pharmacotherapy Education and Research Center, School of Medicine, The University of Texas Health Science Center at San Antonio, 7703 Floyd Curl Dr., MSC-6220, San Antonio, TX 78229, USA

Full list of author information is available at the end of the article
}

pneumonia [2-4]. Lastly, MRSA pneumonia is associated with greater morbidity and mortality than pneumonia caused by other etiologies, possibly due to the virulent and resistant nature of the MRSA pathogen [4]. MRSA accounts for $20-40 \%$ of pneumonia cases that occur after $48 \mathrm{~h}$ into hospital admission and 2-25\% of CO cases overall [4-8].

Previous studies have demonstrated that rapid initiation of appropriate antibiotic therapy is associated with improved survival in hospitalized patients with infections $[9,10]$; therefore, there is a great need for a reliable method to assess CO-MRSA pneumonia risk at admission. Guidelines recommend use of the HCAP criteria to determine need for empiric MRSA therapy, but this definition lacks specificity for CO-MRSA pneumonia and may lead to overuse of broadspectrum antibiotic therapy [2,11]. Finally, prior studies 
have demonstrated that when HCAP patients received guideline-recommended, broad-spectrum therapy (including MRSA therapy), outcomes were no better than when similar patients received alternative antibiotics [12, 13].

Guidance is needed for clinicians to identify those CO-pneumonia patients who might benefit from empiric MRSA therapy.

Recently, Shorr et al. derived a clinical prediction score that stratified patients with CO-pneumonia by their MRSA risk [14]. The risk score consisted of eight variables. Two points were assigned for recent hospitalization or intensive care unit (ICU) admission and one point was assigned for each of the following: age $<30$ or $>79$ years, prior intravenous (IV) antibiotics in last 30 days, dementia, cardiovascular disease, female with diabetes, or recent exposure to a nursing home, long-term care facility, or skilled nursing facility. The total score ranged from 0 to 10 , and patients were stratified into low (0-1), medium (2-5), and high (6-10) risk groups. The CO-MRSA pneumonia prevalence increased from $<10 \%$ in the low-risk group to $>30 \%$ in the high-risk group. The authors concluded that this risk score could help identify those patients at low risk of MRSA, for which MRSA therapy could be spared. They postulated that patients in the high-risk group might benefit from MRSA therapy [14]; however, this has yet to be proven.

The new MRSA risk score could help guide empiric MRSA therapy; however, studies are needed to determine which, if any, of the MRSA risk groups benefit from such therapy. Our primary objective was to compare the effect of MRSA therapy on 30-day patient mortality among CO-pneumonia patients in the three MRSA risk groups (low, medium, and high-risk). Our secondary objective was to determine the association of MRSA risk score with 30-day mortality.

\section{Methods}

This study used administrative data from the Veterans Health Administration (VHA) database. Description of the methods used to build this database have been previously reported $[12,15,16]$. In brief, we performed a retrospective, population-based cohort study using administrative data from the VHA system between fiscal years 2002 and 2007. These data are from over 150 VHA hospitals and 850 VHA outpatient clinics. Data for this study were obtained from the VHA electronic medical record system that includes administrative, clinical, laboratory, and pharmacy data. The Institutional Review Board of the University of Texas Health Science Center at San Antonio and the South Texas Veterans Health Care System Research and Development committee approved this study.

Patients were included if they were $\geq 65$ years of age and had either a primary discharge diagnosis of pneumonia/ influenza (International Classification of Diseases, Ninth Revision, Clinical Modification [ICD-9-CM] codes 480.0-483.99 or 485-487) (Additional file 1) or a secondary discharge diagnosis of pneumonia/influenza plus a primary diagnosis of respiratory failure (ICD-9-CM code 518.81), or sepsis (ICD-9-CM code 038.xx) in fiscal years 2002-2007. If a patient was admitted more than once during the study period, only the first hospitalization was included. Patients were excluded if they did not receive antimicrobial therapy within the first $48 \mathrm{~h}$ of admission.

Baseline demographics were recorded at the time of admission. Antibiotic use was recorded for the first $48 \mathrm{~h}$ of admission. Comorbid conditions were determined using ICD-9-CM codes from outpatient and inpatient care in accordance with the Charlson comorbidity scoring system $[17,18]$. Organ failure included acute and chronic conditions (Additional file 1).

The MRSA risk score variables were defined as: patient age $>79$ years, hospitalization in the past 90 days, (ICU) admission, outpatient (IV) antibiotic therapy within the past 90 days, nursing home resident in the last 90 days, cerebrovascular disease, dementia, and female with diabetes mellitus. These were based on the MRSA risk score developed by Shorr et al. and modified for our database. All of our patients were $\geq 65$ years of age, so the criteria related to age $<30$ years did not apply. Also, recent hospitalization was not limited to stays of $\geq 2$ days, prior IV antibiotic therapy was extended from 30 to 90 days, and ICU admission was not limited to on or before index culture [14]. Each variable contributed one point to the risk score, except for hospitalization in the past 90 days and ICU admission, for which each contributed two points. Patients were stratified into three risk groups based on their risk score: low (0-1), medium (2-5), and high (6-10) [14].

Patients who met study criteria were divided into two groups: the "MRSA therapy" group and the "no MRSA therapy" group. Initial MRSA therapy was defined as the receipt of either vancomycin or linezolid within the first 48 hours of admission. Patients were also categorized based on the receipt of guideline-concordant communityacquired pneumonia (GC-CAP) therapy [19], pseudomonal therapy, and atypical therapy (Table 1). Pneumonia pathogens, including MRSA, were identified using ICD-9CM codes (Additional file 1).

All-cause 30-day patient mortality was the primary study outcome. Previous research has demonstrated that 30-day mortality is more closely associated with pneumonia-related mortality, as compared to 60-day or 90-day mortality [20]. Mortality was assessed using the VHA vital status file, which has been demonstrated to have $98 \%$ exact agreement with the National Death Index, the "gold standard" to determine mortality [21].

\section{Statistical analyses}

All statistical analyses were conducted using JMP $10.0^{\circ}$ (SAS Corp, Cary, NC). Chi-square or Fisher's exact test 
Table 1 Antibiotic therapy definitions

\begin{tabular}{|c|c|}
\hline \multicolumn{2}{|l|}{ MRSA therapy } \\
\hline \multicolumn{2}{|l|}{ - Vancomycin } \\
\hline \multicolumn{2}{|l|}{ • Linezolid } \\
\hline \multicolumn{2}{|l|}{ Guideline-Concordant CAP therapy } \\
\hline Ward patients & ICU patients \\
\hline $\begin{array}{l}\text { - Beta-lactam }{ }^{a} \text { plus (macrolide } \\
\text { or doxycycline) }\end{array}$ & $\begin{array}{l}\text { - Beta-lactam a plus (macrolide }{ }^{b} \text { or } \\
\text { doxycycline) }\end{array}$ \\
\hline - Respiratory fluoroquinolone ${ }^{c}$ & $\begin{array}{l}\text { - Beta-lactam a plus respiratory } \\
\text { fluoroquinolone }\end{array}$ \\
\hline \multicolumn{2}{|l|}{ Pseudomonal therapy } \\
\hline \multicolumn{2}{|c|}{$\begin{array}{l}\text { - Antipseudomonal beta-lactam }{ }^{d} \text { plus antipseudomonal } \\
\text { fluoroquinolone }\end{array}$} \\
\hline \multicolumn{2}{|c|}{ - Antipseudomonal beta-lactam ${ }^{d}$ plus aminoglycoside ${ }^{f}$} \\
\hline \multicolumn{2}{|l|}{ Atypical therapy } \\
\hline \multicolumn{2}{|l|}{ - Macrolide ${ }^{b}$} \\
\hline \multicolumn{2}{|l|}{ - Doxycycline } \\
\hline - Any fluoroquinolone & \\
\hline
\end{tabular}

CAP Community-acquired pneumonia, ICU Intensive care unit

aBeta-lactam includes cefotaxime, ceftriaxone, ampicillin-sulbactam, ertapenum, or aztreonam

${ }^{b}$ Macrolide includes azithromycin, clarithromycin, or erythromycin

${ }^{c}$ Respiratory fluoroquinolone includes moxifloxacin, levofloxacin, or gatifloxacin

${ }^{\mathrm{d}}$ Antipseudomonal beta-lactam includes cefepime, ceftazidime, imipenem-cilastatin, meropenem, piperacillin-tazobactam, or ticarcillin-clavulanate, aztreonam

${ }^{\mathrm{e}}$ Antipseudomonal fluoroquinolone includes ciprofloxacin or levofloxacin

${ }^{f}$ Aminoglycoside includes gentamicin, tobramycin, or amikacin

were used to compare categorical variables between study arms (Table 2). Continuous variables were compared using the Wilcoxon Rank Sum test (Table 2). For bivariate statistical tests, we defined significance as a two-tailed alpha $\leq 0.0001$ to avoid spurious associations in this large patient cohort.

Separate multivariable logistic regression models were constructed to examine if MRSA therapy was associated with 30-day mortality in the overall population and each of the three MRSA risk groups. The dependent variable was 30-day patient mortality, and the independent variable was MRSA therapy versus no MRSA therapy. Covariates included all unbalanced characteristics that were significant in the bivariate analysis comparing 30-day mortality versus no 30-day mortality (Additional file 2). Finally, MRSA culture-positivity was also entered into the model. Adjusted odds ratios (aORs) and $95 \%$ confidence intervals (95\% CIs) were calculated; those $95 \%$ CIs that did not cross one were considered to be statistically significant.

A few of the variables were excluded from the model because of collinearity. Collinearity was determined through theoretical relations for select variables. For instance, most patients on hemodialysis also had renal failure; therefore, hemodialysis was chosen for the model. Likewise, most patients who had diabetes mellitus also received anti-diabetic medications; therefore, the anti- diabetic medications variable was chosen for the model. The Charlson score and the "any organ failure" variables were excluded from the model because individual comorbidities and organ failures were already included in the model. Individual MRSA risk score variables were also excluded from the model because we ran separate multivariable models for the three risk groups, and these individual characteristics were used to define those risk groups.

The final list of 25 covariates included: patient age, race, Hispanic ethnicity, myocardial infarction, heart failure, chronic obstructive pulmonary disease (COPD), liver disease, renal disease, neoplastic disease, cardiovascular medications, anti-diabetic medications, inhaled corticosteroids, pulmonary medications, vasopressors, invasive and non-invasive mechanical ventilation, respiratory failure, cardiovascular failure, neurological failure, renal failure, hematological failure, hepatic failure, GC-CAP therapy, pseudomonal therapy, atypical therapy, and MRSA culturepositivity.

\section{Results}

\section{Overall population}

Baseline patient characteristics are shown in Table 2. A total of 80,330 patients met inclusion criteria, with $36 \%$ in the MRSA therapy group and $64 \%$ in the no MRSA therapy group. Patients were predominately elderly (median age 78 years), white $(81 \%)$ men $(98 \%)$. Age $>79$ was the most common MRSA risk factor (44\%), followed by hospitalization in the past 90 days (28\%) and ICU admission $(21 \%)$. There were very few women with diabetes mellitus $(0.4 \%)$ in this population. The median MRSA risk score was 1 (interquartile range [IQR] 0-3). The majority of patients were classified as either low (51\%) or medium (47\%) risk, with only $2 \%$ classified as high-risk. None of the patients scored above an 8 on the MRSA risk score.

The median (IQR) Charlson score was 2 (1-4), and common comorbidities included COPD (49\%), diabetes (31\%), heart failure (26\%), and neoplastic disease (25\%). The most commonly used medications within 90 days prior to admission included cardiovascular medications (67\%) and pulmonary medications (35\%). Organ failure occurred in $32 \%$ of patients. Finally, most patients received atypical (75 \%) and GC-CAP (64\%) therapy.

\section{Baseline characteristics}

Patient age and sex were similar between MRSA therapy and no MRSA therapy groups. A lower proportion of white patients received MRSA therapy, while higher proportions of black and Hispanic patients received MRSA therapy.

Charlson scores were higher in the MRSA therapy group and these patients had a higher prevalence of heart failure, renal disease, diabetes, and neoplastic disease. In addition, the MRSA therapy group had a higher prevalence of vasopressor use, mechanical ventilation, hemodialysis, organ 
Table 2 Baseline characteristics grouped by MRSA therapy

\begin{tabular}{|c|c|c|c|c|}
\hline & Overall $(n=80,330)$ & MRSA therapy $(n=29,254)$ & No MRSA therapy $(n=51,076)$ & $P$-value \\
\hline Patient age (years), median (IQR) & $78(72-83)$ & $78(73-83)$ & $77(72-83)$ & 0.1016 \\
\hline Male, \% & 98.3 & 98.3 & 98.3 & 0.6847 \\
\hline \multicolumn{5}{|l|}{ Race, \% } \\
\hline White & 81.1 & 79.1 & 82.3 & $<0.0001$ \\
\hline Black & 13.1 & 15.4 & 11.7 & $<0.0001$ \\
\hline Other & 5.8 & 5.5 & 6.0 & $<0.0001$ \\
\hline Hispanic ethnicity, \% & 7.0 & 8.0 & 6.4 & $<0.0001$ \\
\hline \multicolumn{5}{|l|}{ MRSA risk score variables, \% (1 point, unless noted) } \\
\hline Age $>79$ & 43.8 & 44.0 & 43.7 & 0.3867 \\
\hline Hospitalization in the past 90 days (2 points) & 27.8 & 33.7 & 24.5 & $<0.0001$ \\
\hline Intensive care unit admission ( 2 points) & 21.1 & 29.3 & 16.4 & $<0.0001$ \\
\hline Outpatient IV antibiotic therapy in past 90 days & 4.9 & 5.3 & 4.7 & 0.0001 \\
\hline Nursing home resident in last 90 days & 1.0 & 1.1 & 0.9 & 0.0028 \\
\hline Cerebrovascular disease & 18.1 & 19.6 & 17.3 & $<0.0001$ \\
\hline Dementia & 5.2 & 5.8 & 4.9 & $<0.0001$ \\
\hline Female with diabetes mellitus & 0.4 & 0.4 & 0.4 & 0.8129 \\
\hline MRSA risk score, median (IQR) & $1(0-3)$ & $2(1-3)$ & $1(0-2)$ & $<0.0001$ \\
\hline Low $(0-1), \%$ & 51.4 & 41.6 & 57.0 & $<0.0001$ \\
\hline Medium (2-5), \% & 47.3 & 56.4 & 42.1 & $<0.0001$ \\
\hline High (6-10), \% & 1.3 & 2.1 & 0.9 & $<0.0001$ \\
\hline Charlson comorbidity score, median (IQR) & $2(1-4)$ & $3(1-4)$ & $2(1-4)$ & $<0.0001$ \\
\hline \multicolumn{5}{|l|}{ Comorbid conditions, \% } \\
\hline Myocardial infarction & 7.2 & 7.6 & 7.0 & 0.0020 \\
\hline Heart failure & 25.9 & 27.0 & 25.3 & $<0.0001$ \\
\hline Chronic obstructive pulmonary disease & 48.7 & 45.5 & 50.5 & $<0.0001$ \\
\hline Liver disease & 1.3 & 1.5 & 1.2 & 0.0035 \\
\hline Renal disease & 14.1 & 17.2 & 12.3 & $<0.0001$ \\
\hline Diabetes & 30.5 & 31.8 & 29.8 & $<0.0001$ \\
\hline Neoplastic disease & 25.2 & 26.2 & 24.7 & $<0.0001$ \\
\hline HIV/AIDS & 0.2 & 0.3 & 0.2 & 0.0961 \\
\hline \multicolumn{5}{|l|}{ Medication use within 90 days, \% } \\
\hline Cardiovascular medications & 66.5 & 64.9 & 67.3 & $<0.0001$ \\
\hline Anti-diabetic medications & 22.2 & 22.6 & 22.1 & 0.0852 \\
\hline Inhaled corticosteroids & 21.1 & 18.9 & 22.4 & $<0.0001$ \\
\hline Systemic corticosteroids ${ }^{a}$ & 22.2 & 21.8 & 22.5 & 0.0217 \\
\hline Pulmonary medications & 34.8 & 31.4 & 36.8 & $<0.0001$ \\
\hline Vasopressors, \% & 10.2 & 15.2 & 7.2 & $<0.0001$ \\
\hline Invasive mechanical ventilation, \% & 11.1 & 16.3 & 8.2 & $<0.0001$ \\
\hline Noninvasive mechanical ventilation, \% & 4.0 & 5.7 & 3.1 & $<0.0001$ \\
\hline Hemodialysis, \% & 18.3 & 22.7 & 15.7 & $<0.0001$ \\
\hline \multicolumn{5}{|l|}{ Organ failure, \% } \\
\hline Any organ failure, $\%$ & 32.2 & 41.8 & 26.7 & $<0.0001$ \\
\hline Respiratory & 14.4 & 19.4 & 11.5 & $<0.0001$ \\
\hline Cardiovascular & 9.7 & 13.0 & 7.8 & $<0.0001$ \\
\hline
\end{tabular}


Table 2 Baseline characteristics grouped by MRSA therapy (Continued)

\begin{tabular}{|c|c|c|c|c|}
\hline Neurological & 2.5 & 3.3 & 2.0 & $<0.0001$ \\
\hline Renal & 20.1 & 26.8 & 16.2 & $<0.0001$ \\
\hline Hematologic & 4.1 & 5.5 & 3.3 & $<0.0001$ \\
\hline Hepatic & 0.7 & 0.9 & 0.6 & $<0.0001$ \\
\hline \multicolumn{5}{|l|}{ Antibiotic therapy, \% } \\
\hline Guideline-concordant CAP therapy & 64.1 & 64.9 & 63.6 & 0.0001 \\
\hline Pseudomonal therapy & 17.5 & 31.1 & 9.7 & $<0.0001$ \\
\hline Atypical therapy & 75.2 & 83.6 & 70.5 & $<0.0001$ \\
\hline
\end{tabular}

failure, GC-CAP therapy, pseudomonal therapy, and atypical therapy. In contrast, the no MRSA therapy group had a higher prevalence of COPD and were more likely to receive cardiovascular medications, inhaled corticosteroids, and pulmonary medications in the last 90 days.

\section{Bacterial pathogens}

The prevalence of bacterial pathogens is shown in Table 3. Staphylococcus aureus was the most commonly isolated pathogen and the majority of those isolates were MRSA. Streptococcus pneumoniae was the second most common pathogen. The MRSA therapy group had more patients who were culture-positive and had higher rates of Staphylococcus aureus, MRSA, Pseudomonas spp., and most other gram-negative pathogens, except for Haemophilus influenzae.

\section{Patient mortality}

The overall 30-day patient mortality rate was $20 \%$, and the unadjusted 30-day mortality increased from the low (11\%), medium (27\%), and high (48\%) risk groups $(p<0.0001)$. In addition, an increase in unadjusted 30-day mortality was observed with each additional point of the MRSA risk score (Fig. 1). Unadjusted 30-day mortality was higher among patients who received MRSA therapy in the low-

Table 3 Bacterial pathogen distribution grouped by MRSA therapy

\begin{tabular}{|c|c|c|c|c|}
\hline & Overall $(n=80,330)$ & MRSA therapy $(n=29,254)$ & No MRSA therapy $(n=51,076)$ & $P$-value ${ }^{*}$ \\
\hline Organism identified, \% & 10.3 & 13.9 & 8.2 & $<0.0001$ \\
\hline Single organism identified & 9.3 & 12.1 & 7.7 & $<0.0001$ \\
\hline Multiple organisms identified & 1.0 & 1.9 & 0.5 & $<0.0001$ \\
\hline \multicolumn{5}{|l|}{ Gram-positive pathogens, \% } \\
\hline Streptococcus pneumoniae & 2.8 & 2.7 & 2.9 & 0.0571 \\
\hline Streptococcus, other & 0.4 & 0.5 & 0.4 & 0.0270 \\
\hline Staphylococcus aureus & 5.1 & 8.7 & 3.1 & $<0.0001$ \\
\hline MRSA & 3.3 & 5.1 & 2.3 & $<0.0001$ \\
\hline \multicolumn{5}{|l|}{ Gram-negative pathogens, $\%$} \\
\hline Klebsiella pneumoniae & 0.7 & 1.0 & 0.6 & $<0.0001$ \\
\hline Pseudomonas spp. & 1.5 & 1.9 & 1.2 & $<0.0001$ \\
\hline Haemophilus influenzae & 0.8 & 0.5 & 0.9 & $<0.0001$ \\
\hline Escherichia coli & 0.2 & 0.4 & 0.2 & $<0.0001$ \\
\hline Other gram-negatives & 0.5 & 0.6 & 0.4 & 0.0007 \\
\hline \multicolumn{5}{|l|}{ Atypical pathogens, $\%$} \\
\hline Mycoplasma pneumoniae & $<0.1$ & $<0.1$ & 0.1 & 0.0839 \\
\hline Legionella spp. & 0.1 & 0.2 & 0.1 & 0.2029 \\
\hline Chlamydia spp. & $<0.1$ & $<0.1$ & $<0.1$ & 0.7561 \\
\hline Anaerobes, \% & 0.1 & 0.1 & 0.1 & 0.0139 \\
\hline
\end{tabular}

MRSA Methicillin-resistant Staphylococcus aureus

* Comparison between "MRSA therapy" versus "no MRSA therapy" groups 


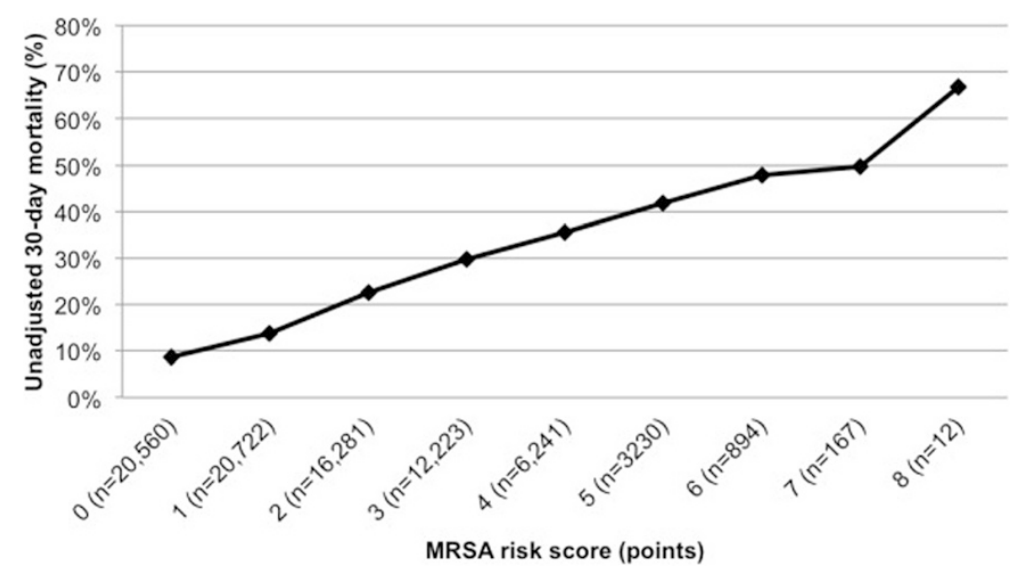

Fig. 1 Unadjusted 30-day patient mortality by MRSA risk score. MRSA Methicillin-resistant Staphylococcus aureus

risk group (15\% versus $9 \% ; p<0.0001$ ), but lower in the high-risk group (40\% versus $58 \%$; $p<0.0001$ ) (Fig. 2). After adjustment for potential confounders, MRSA therapy was associated with higher 30-day mortality in the low-risk group (aOR 1.47; 95 \% CI 1.37-1.58) and lower 30-day mortality in the high-risk group (aOR 0.57; $95 \%$ CI 0.42-0.77) (Fig. 2).

More than 40 characteristics were compared among patients who survived and those who died within 30-days of hospital discharge (Table 4). The multivariable regression analysis revealed that several of those variables were independently associated with 30-day mortality (Table 5). Risk factors varied for low, medium, and high-risk groups.

\section{Discussion}

CO-MRSA pneumonia is an evolving problem, and clinicians need strategies to determine appropriate candidates for empiric MRSA therapy. Shorr et al. developed a risk score to specifically identify CO-pneumonia patients at risk for MRSA infection [14]. Using a MRSA risk score similar to Shorr et al., our study demonstrates a survival advantage for CO-pneumonia among high-risk patients who received initial MRSA therapy. The number needed to treat with initial MRSA therapy to save one life in the high-risk group was 5 . This survival advantage was not present for patients in the low and medium-risk groups.

Our study also supports the notion that the MRSA risk score might be a better alternative to guide empiric MRSA therapy in patients with CO-pneumonia as compared to the HCAP criteria. Several studies have demonstrated that the HCAP criteria have low specificity for MRSA pneumonia, and that grouping risk factors for MRSA with other gram-negative MDR pathogens may lead to inappropriate treatment [11, 22-25]. Recently, Chalmers et al. conducted a meta-analysis of 24 studies that compared HCAP and CAP cohorts. The study concluded that the ability for the HCAP criteria to appropriately identify patients with MDR pathogens (including MRSA) was low and did not meet

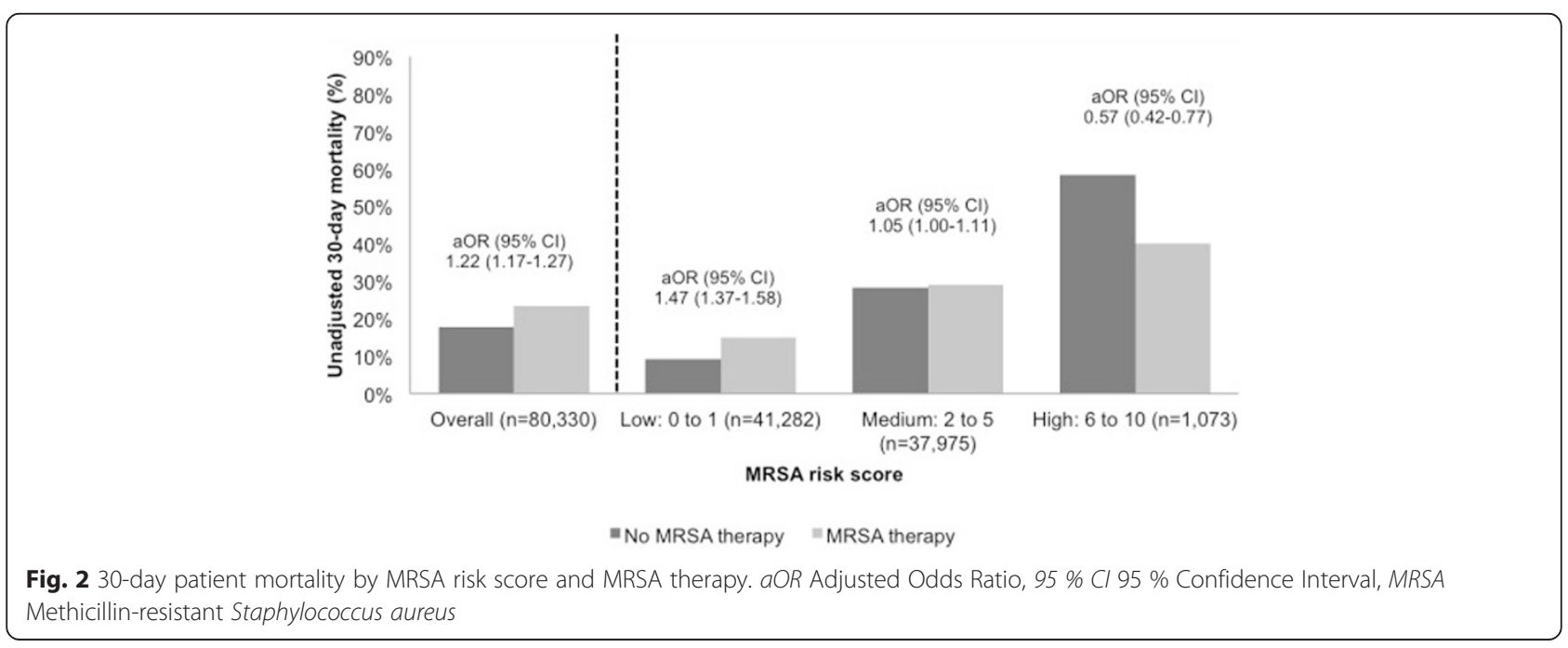


Table 4 Baseline characteristics grouped by 30-day patient mortality

\begin{tabular}{|c|c|c|c|c|}
\hline & Overall $(n=80,330)$ & 30-day mortality $(n=15,909)$ & No 30-day mortality $(n=64,421)$ & $P$-value \\
\hline Patient age (years), median (IQR) & $78(72-83)$ & 79 (74-84) & $77(72-82)$ & $<0.0001$ \\
\hline Male, \% & 98.3 & 98.5 & 98.2 & 0.0100 \\
\hline \multicolumn{5}{|l|}{ Race, \% } \\
\hline White & 81.1 & 77.3 & 82.0 & $<0.0001$ \\
\hline Black & 13.1 & 14.9 & 12.6 & $<0.0001$ \\
\hline Other & 5.8 & 7.8 & 5.3 & $<0.0001$ \\
\hline Hispanic ethnicity, \% & 7.0 & 7.9 & 6.8 & $<0.0001$ \\
\hline \multicolumn{5}{|l|}{ MRSA risk score variables, \% (1 point, unless noted) } \\
\hline Age $>79$ & 43.8 & 51.0 & 42.0 & $<0.0001$ \\
\hline Hospitalization in the past 90 days (2 points) & 27.8 & 40.7 & 24.7 & $<0.0001$ \\
\hline Intensive care unit admission ( 2 points) & 21.1 & 42.0 & 16.0 & $<0.0001$ \\
\hline Outpatient IV antibiotic therapy in past 90 days & 4.9 & 6.0 & 4.7 & $<0.0001$ \\
\hline Nursing home resident in last 90 days & 1.0 & 0.8 & 1.0 & 0.0292 \\
\hline Cerebrovascular disease & 18.1 & 20.0 & 17.7 & $<0.0001$ \\
\hline Dementia & 5.2 & 6.7 & 4.9 & $<0.0001$ \\
\hline Female with diabetes mellitus & 0.4 & 0.4 & 0.4 & 0.8881 \\
\hline MRSA risk score, median (IQR) & $1(0-3)$ & $2(1-2)$ & $1(0-2)$ & $<0.0001$ \\
\hline Low $(0-1), \%$ & 51.4 & 28.4 & 57.1 & $<0.0001$ \\
\hline Medium (2-5), \% & 47.3 & 68.4 & 42.1 & $<0.0001$ \\
\hline High $(6-10), \%$ & 1.3 & 3.25 & 0.9 & $<0.0001$ \\
\hline Charlson comorbidity score, median (IQR) & $2(1-4)$ & $3(1-5)$ & $2(1-4)$ & $<0.0001$ \\
\hline \multicolumn{5}{|l|}{ Comorbid conditions, \% } \\
\hline Myocardial infarction & 7.3 & 8.7 & 6.9 & $<0.0001$ \\
\hline Heart failure & 25.9 & 28.2 & 25.4 & $<0.0001$ \\
\hline Chronic obstructive pulmonary disease & 48.7 & 41.7 & 50.4 & $<0.0001$ \\
\hline Liver disease & 1.3 & 2.3 & 1.1 & $<0.0001$ \\
\hline Renal disease & 14.1 & 16.7 & 13.4 & $<0.0001$ \\
\hline Diabetes & 30.5 & 30.3 & 30.5 & 0.5493 \\
\hline Neoplastic disease & 25.2 & 31.1 & 23.8 & $<0.0001$ \\
\hline HIV/AIDS & 0.2 & 0.2 & 0.2 & 0.2145 \\
\hline \multicolumn{5}{|l|}{ Medication use within 90 days, $\%$} \\
\hline Cardiovascular medications & 66.5 & 60.0 & 68.0 & $<0.0001$ \\
\hline Anti-diabetic medications & 22.2 & 20.0 & 22.8 & $<0.0001$ \\
\hline Inhaled corticosteroids & 21.1 & 14.8 & 22.7 & $<0.0001$ \\
\hline Systemic corticosteroids ${ }^{a}$ & 22.2 & 21.1 & 22.5 & 0.0002 \\
\hline Pulmonary medications & 34.8 & 27.8 & 36.6 & $<0.0001$ \\
\hline Vasopressors, \% & 10.2 & 26.9 & 6.0 & $<0.0001$ \\
\hline Invasive mechanical ventilation, \% & 11.1 & 26.8 & 7.3 & $<0.0001$ \\
\hline Noninvasive mechanical ventilation, \% & 4.0 & 7.1 & 3.3 & $<0.0001$ \\
\hline Hemodialysis, \% & 18.3 & 23.5 & 17.0 & $<0.0001$ \\
\hline \multicolumn{5}{|l|}{ Organ failure, \% } \\
\hline Any organ failure, $\%$ & 32.2 & 57.8 & 25.9 & $<0.0001$ \\
\hline Respiratory & 14.4 & 32.6 & 9.9 & $<0.0001$ \\
\hline Cardiovascular & 9.7 & 22.1 & 6.7 & $<0.0001$ \\
\hline
\end{tabular}


Table 4 Baseline characteristics grouped by 30-day patient mortality (Continued)

\begin{tabular}{|c|c|c|c|c|}
\hline Neurological & 2.5 & 4.2 & 2.0 & $<0.0001$ \\
\hline Renal & 20.1 & 35.3 & 16.3 & $<0.0001$ \\
\hline Hematologic & 4.1 & 8.3 & 3.0 & $<0.0001$ \\
\hline Hepatic & 0.7 & 2.0 & 0.3 & $<0.0001$ \\
\hline \multicolumn{5}{|l|}{ Antibiotic therapy, \% } \\
\hline MRSA therapy & 36.4 & 42.9 & 34.8 & $<0.0001$ \\
\hline Guideline-concordant CAP therapy & 64.1 & 38.6 & 70.4 & $<0.0001$ \\
\hline Pseudomonal therapy & 17.5 & 23.8 & 15.9 & $<0.0001$ \\
\hline Atypical therapy & 75.2 & 54.3 & 80.4 & $<0.0001$ \\
\hline
\end{tabular}

the threshold for clinical use [26]. In addition, most studies evaluating the utility of HCAP criteria to guide broad-spectrum therapy, including MRSA therapy, have not demonstrated improved mortality with guidelineconcordant therapy $[12,13]$. In a large Canadian cohort study, Grenier et al. were unable to demonstrate a 30-day mortality benefit in HCAP patients treated with guidelineconcordant antimicrobials [13]. In 2012, Madaras-Kelly et al. evaluated guideline-endorsed HCAP regimens by stratifying patients based on risk for MDR pathogens [27]. This study demonstrated that patients who were at higher risk for MDR pathogens had a survival benefit if they were treated with guideline-endorsed therapies; however, those with low risk for MDR pathogens who received guidelineendorsed therapies had significantly higher mortality.

One advantage of the MRSA risk score is weighting of important risk factors. The MRSA risk score assigns more weight (2 points each) to patients with recent hospitalization and severe pneumonia requiring ICU admission compared to the other risk factors (1 point each). In a recent study stratifying risk factors for MDR pathogens in CO-pneumonia patients, recent hospitalization and severe pneumonia were found to be independent predictors of mortality [28]. This highlights a key feature of the MRSA risk score, because patients with either of those two risk factors cannot be classified into the low-risk group.

Recently, Minejima et al. compared a smaller cohort of CO-pneumonia patients with culture-proven MRSA pneumonia and non-MRSA pneumonia. They found $37 \%$ of patients with non-MRSA pneumonia met HCAP criteria and $28 \%$ received unnecessary MRSA therapy. Had the MRSA risk score been applied, unwarranted MRSA therapy could have been decreased by $20 \%$ in low-risk patients without MRSA pneumonia [29]. Our findings further support the idea that the new MRSA risk score can help identify low-risk CO-pneumonia patients who are unlikely to benefit from empiric MRSA therapy.
Our study has important limitations. First, prior studies have identified risk factors associated with CO-MRSA pneumonia that were not included in the MRSA risk score. These include: MRSA infection in the past year, known MRSA colonization, necrotizing or cavitary pneumonia, severity-of-illness scores, and preceding or concurrent influenza [19, 29-36]. Similarly, there are patient and provider characteristics that were not included in our analyses that might affect patient outcomes. These include specific antimicrobial medications received, non-antimicrobial medications, pathogens, antimicrobial susceptibilities, patient functional status and clinical presentation, and provider preferences. The study inclusion dates might not provide a good reflection of current prescribing practices for CO-pneumonia; however it is important to note that we sorted the patients into guideline-concordant and discordant groups according to the recommendations in the most recent CAP guidelines. We evaluated the impact of guideline-concordant therapy, pseudomonal therapy, and atypical therapy in our multivariable models; however, we did not study specific medications and combinations, some of which might be associated with better outcomes. The data source included a predominately elderly male population. This population had higher 30-day mortality than that typically seen in non-VA populations, which could limit the generalizability of our results. In addition, the older age of the population precluded our ability to assess Shorr's MRSA risk criteria for age $<30$ years. Other changes in our variable definitions might also have affected outcomes. We altered the prior IV antibiotic use criteria to include use in the prior 90 days, rather than 30 days. This is likely to result in a larger proportion of patients who meet this criterion, and might bias the population to higher MRSA risk scores.

ICD-9-CM codes were used to identify pneumonia patients, pathogens, and baseline characteristics. This approach could potentially lead to misclassification bias, or underestimate the true prevalence of the pathogens, and 
Table 5 Risk factors for 30-day patient mortality grouped by MRSA risk score

\begin{tabular}{|c|c|c|c|c|}
\hline \multirow[b]{2}{*}{ Risk score } & \multicolumn{4}{|c|}{ Adjusted Odds ratio (95 \% confidence interval) } \\
\hline & All $(n=80,330)$ & $0-1(n=41,282)$ & $2-5(n=37,975)$ & $6-10(n=1,073)$ \\
\hline MRSA therapy & $1.22(1.17-1.27)$ & $1.47(1.37-1.58)$ & $1.05(1.00-1.11)$ & $0.57(0.42-0.77)$ \\
\hline Age (1-year increments) & $1.05(1.04-1.05)$ & $1.05(1.05-1.06)$ & $1.04(1.03-1.04)$ & $1.05(1.02-1.08)$ \\
\hline Race & $0.99(0.94-1.05)$ & $0.95(0.86-1.06)$ & $0.98(0.91-1.05)$ & $1.18(0.85-1.64)$ \\
\hline Hispanic ethnicity & $0.85(0.79-0.92)$ & $0.84(0.73-0.96)$ & $0.86(0.79-0.95)$ & $0.79(0.47-1.32)$ \\
\hline \multicolumn{5}{|l|}{ Comorbid conditions } \\
\hline Myocardial infarction & $1.14(1.06-1.23)$ & $1.11(0.94-1.30)$ & $1.05(0.97-1.14)$ & $1.06(0.74-1.51)$ \\
\hline Heart failure & $1.16(1.11-1.21)$ & $1.07(0.97-1.17)$ & $1.11(1.05-1.18)$ & $1.16(0.88-1.53)$ \\
\hline COPD & $0.96(0.91-1.00)$ & $0.92(0.85-1.00)$ & $0.95(0.89-1.01)$ & $1.30(0.96-1.77)$ \\
\hline Liver disease & $1.79(1.54-2.08)$ & $1.34(0.97-1.87)$ & $1.80(1.50-2.14)$ & $3.46(1.08-13.51)$ \\
\hline Renal disease & $0.98(0.93-1.04)$ & $0.91(0.81-1.02)$ & $0.96(0.90-1.03)$ & $1.12(0.83-1.53)$ \\
\hline Neoplastic disease & $1.54(1.47-1.60)$ & $1.57(1.46-1.69)$ & $1.48(1.40-1.56)$ & $1.18(0.87-1.59)$ \\
\hline \multicolumn{5}{|l|}{ Medication use, by class } \\
\hline Cardiovascular medications & $0.72(0.69-0.75)$ & $0.60(0.56-0.64)$ & $0.78(0.73-0.82)$ & $0.91(0.66-1.24)$ \\
\hline Anti-diabetic medications & $0.92(0.87-0.96)$ & $0.78(0.71-0.86)$ & $0.95(0.90-1.01)$ & $0.95(0.70-1.29)$ \\
\hline Inhaled corticosteroids & $0.76(0.72-0.81)$ & $0.73(0.65-0.81)$ & $0.80(0.74-0.86)$ & $1.13(0.77-1.65)$ \\
\hline Pulmonary medications & $1.00(0.94-1.05)$ & $0.93(0.85-1.03)$ & $1.03(0.97-1.11)$ & $0.68(0.48-0.97)$ \\
\hline Vasopressors & $1.81(1.70-1.93)$ & $3.00(2.49-3.61)$ & $1.66(1.55-1.79)$ & $1.52(1.12-2.04)$ \\
\hline \multicolumn{5}{|l|}{ Mechanical ventilation } \\
\hline Invasive & $1.08(1.01-1.16)$ & $2.48(1.98-3.10)$ & $1.02(0.95-1.10)$ & $0.92(0.65-1.29)$ \\
\hline Noninvasive & $1.16(1.06-1.27)$ & $2.03(1.60-2.57)$ & $1.03(0.94-1.13)$ & $1.22(0.80-1.86)$ \\
\hline \multicolumn{5}{|l|}{ Organ failure } \\
\hline Respiratory & $2.48(2.34-2.63)$ & $3.84(3.45-4.29)$ & $2.03(1.90-2.18)$ & $1.47(1.06-2.04)$ \\
\hline Cardiovascular & $1.91(1.80-2.02)$ & $1.90(1.68-2.15)$ & $1.81(1.69-1.94)$ & $1.36(1.02-1.82)$ \\
\hline Neurological & $1.53(1.38-1.71)$ & $1.64(1.33-2.02)$ & $1.40(1.23-1.58)$ & $1.92(1.03-3.65)$ \\
\hline Renal & $1.58(1.51-1.66)$ & $1.77(1.62-1.94)$ & $1.46(1.38-1.55)$ & $1.15(0.87-1.51)$ \\
\hline Hematologic & $1.65(1.52-1.80)$ & $1.97(1.67-2.32)$ & $1.53(1.39-1.69)$ & $1.18(0.73-1.90)$ \\
\hline Hepatic & $2.54(2.08-3.11)$ & $3.21(2.05-5.00)$ & $2.38(1.90-2.99)$ & $0.66(0.20-2.16)$ \\
\hline \multicolumn{5}{|l|}{ Antibiotic therapy } \\
\hline GC-CAP therapy & $0.67(0.63-0.70)$ & $0.60(0.54-0.67)$ & $0.73(0.69-0.79)$ & $0.73(0.50-1.08)$ \\
\hline Pseudomonal therapy & $1.25(1.19-1.32)$ & $1.68(1.53-1.85)$ & $1.05(0.99-1.12)$ & $0.76(0.54-1.06)$ \\
\hline Atypical therapy & $0.45(0.43-0.48)$ & $0.59(0.53-0.66)$ & $0.46(0.43-0.49)$ & $0.75(0.52-1.08)$ \\
\hline MRSA culture positivity & $0.66(0.59-0.74)$ & $0.58(0.45-0.73)$ & $0.66(0.58-0.75)$ & $0.56(0.31-0.99)$ \\
\hline
\end{tabular}

Bold indicates statistical significance; MRSA Methicillin-resistant Staphylococcus aureus; Race was ordered as black versus nonblack; COPD Chronic obstructive pulmonary disease, GC-CAP Guideline-concordant community-acquired pneumonia

cannot be considered equivalent to a medical chart review. There was no MRSA pneumonia code during the time these patients were treated for pneumonia; therefore, MRSA pneumonia was defined as a patient with a pneumonia ICD-9 code plus a general MRSA ICD-9 code that was not specific for MRSA pneumonia. Because of this, the general MRSA code could represent some other infectious process occurring simultaneously with pneumonia and not necessarily MRSA pneumonia. Furthermore, the V09.0 code is an imprecise way to identify MRSA infections, in general [37]. However, there is evidence to suggest that the code more accurately identifies MRSA infections in community-onset patients (like the ones in this study) than in hospital-onset patients [38]. In the absence of a single code that couples disease with pathogen, many large databases, like ours, rely on existing ICD9 codes, like the V09.0 MRSA code plus pneumonia codes, for case definitions. A published study from another group [39], and one from our own group [40], demonstrate examples where investigators have 
used the V09.0 code to identify patients with MRSA infections.

Also, it is possible that patients in the MRSA low-risk group did worse because of the greater prevalence of some other problem pathogen, for which anti-MRSA antibiotics have no activity. Reliance on ICD-9 codes for pathogen identification resulted in a lower rate of pathogen identification, so this is not the best study design to identify problem pathogens. Since this is an observational study, the impact of MRSA therapy would be better assessed if the propensity score of MRSA therapy was adjusted for in the analysis, so that the bias due to observed confounding (baseline covariates that were associated with MRSA use) could be minimized. Also, for unobserved confounding, an instrumental variable approach would further reduce the bias due to unobserved confounding. However, our analyses did not consider the propensity score nor instrumental variable adjustments due to the limited baseline data available from the electronic medical record database and lack of a credible instrumental variable that can be identified in the literature.

\section{Conclusions}

This study demonstrated improved survival with initial MRSA therapy in high-risk CO-pneumonia patients. The MRSA risk score might help spare MRSA therapy for only those patients who are likely to benefit.

\section{Additional files}

Additional file 1: ICD-9-CM codes for patient variables and bacterial pathogens. (DOCX $25 \mathrm{~kb}$ )

Additional file 2: List of medications used within 90 days of admission, by class. (DOCX $24 \mathrm{~kb}$ )

\section{Abbreviations}

CO: Community-onset; MRSA: Methicillin-resistant Staphylococcus aureus; CAP: Community-acquired pneumonia; HCAP: Healthcare-associated pneumonia; MDR: Multidrug-resistant; VHA: Veterans Health Administration; ICD-9-CM: International Classification of Diseases, Ninth Revision, Clinical Modification codes; ICU: Intensive care unit; IV: Intravenous; GC: Guidelineconcordant; aOR: Adjusted odds ratio; Cl: Confidence interval; COPD: Chronic obstructive pulmonary disease; IQR: Interquartile range.

\section{Competing interests}

Dr. Frei has received research grants, to his institution, for investigator-initiated cancer and infectious diseases research, from Bristol Myers Squibb, Forest, Ortho-McNeil Janssen, and Pfizer in the past 3 years. Mr. Koeller has received research grants, to his institution, for investigator-initiated cancer research, from Bristol Myers Squibb in the past 3 years.

\section{Authors' contributions}

Study concept and design: CRF, BFT. Statistical analysis: CRF, BFT. Interpretation of data: RTA, CRF, JK, GCL, KRR, BFT. Drafting of the manuscript: CRF, BFT. Critical revision of the manuscript for important intellectual content: All authors. Study supervision: CRF. All authors read and approved the final manuscript.

\section{Acknowledgements}

This study was supported by a grant from the National Institutes of Health $(\mathrm{NIH})$ /National Institute of Nursing Research (R01NR010828). Dr. Frei was supported in part by a NIH Clinical Research Scholar (KL2) career development award (National Center for Research Resources 5KL2 RR025766 [2010-2012] and National Center for Advancing Translational Sciences 8KL2 TR000118 [2013]). Dr. Mortensen was supported in part by a grant from the Agency for Healthcare Research and Quality (R24 HS022418) and the University of Texas Southwestern Center for Patient-Centered Outcomes Research. This material is the result of work supported with resources and the use of facilities at the South Texas Veterans Health Care System. The funding sources had no role in the design and conduct of the study; collection, management, analysis, and interpretation of the data; preparation, review, or approval of the manuscript; and decision to submit the manuscript for publication. The views expressed in this article are those of the authors and do not necessarily represent the views of the Department of Veterans Affairs or the National Institutes of Health. Finally, the co-authors would like to acknowledge the assistance of Stefan Allen and Ashley Pechal (pharmacy students at The University of Texas at Austin College of Pharmacy, who assisted the co-authors with literature searches during part of the peer review process).

\section{Author details}

${ }^{1}$ St. Louis College of Pharmacy, St. Louis, MO, USA. ${ }^{2}$ Pharmacotherapy Division, College of Pharmacy, The University of Texas at Austin, Austin, TX, USA. ${ }^{3}$ Pharmacotherapy Education and Research Center, School of Medicine, The University of Texas Health Science Center at San Antonio, 7703 Floyd Curl Dr., MSC-6220, San Antonio, TX 78229, USA. ${ }^{4}$ Feik School of Pharmacy, University of the Incarnate Word, San Antonio, TX, USA. ${ }^{5}$ South Texas Veterans Health Care System, San Antonio, TX, USA. ${ }^{6}$ Department of Epidemiology and Biostatistics, University of Texas Health Science Center, San Antonio, TX, USA. ${ }^{7}$ The VA North Texas Health Care System, Dallas, TX USA. ${ }^{8}$ The University of Texas Southwestern Medical Center, Dallas, TX, USA.

Received: 1 November 2014 Accepted: 10 September 2015

Published online: 18 September 2015

\section{References}

1. Murphy SL, Xu J, Kochanek KD. Deaths: final data for 2010. Natl Vital Stat Rep. 2013;61:1-118.

2. American Thoracic Society, Infectious Diseases Society of America. Guidelines for the management of adults with hospital-acquired, ventilator-associated, and healthcare-associated pneumonia. Am J Respir Crit Care Med. 2005;171:388-416.

3. Friedman ND, Kaye KS, Stout JE, McGarry SA, Trivette SL, Briggs JP, et al. Health care-associated bloodstream infections in adults: a reason to change the accepted definition of community-acquired infections. Ann Intern Med. 2002;137(10):791-7.

4. Rubinstein E, Kollef MH, Nathwani D. Pneumonia caused by methicillinresistant Staphylococcus aureus. Clin Infect Dis. 2008;46(Supplement 5):S378-85.

5. Kollef MH, Shorr AF, Tabak YP, Gupta V, Liu LZ, Johannes RS. Epidemiology and outcomes of health-care-associated pneumonia: results from a large US database of culture-positive pneumonia. Chest. 2005;128(6):3854-62.

6. Micek ST, Kollef KE, Reichley RM, Roubinian N, Kollef MH. Health care-associated pneumonia and community-acquired pneumonia: a single-center experience. Antimicrob Agents Chemother. 2007:51(10):3568-73.

7. Chalmers JD, Taylor JK, Singanayagam A, Fleming GB, Akram AR, Mandal P, et al. Epidemiology, antibiotic therapy, and clinical outcomes in health careassociated pneumonia: a UK cohort study. Clin Infect Dis. 2011;53(2):107-13.

8. Moran GJ, Krishnadasan A, Gorwitz RJ, Fosheim GE, Albrecht V, Limbago B, et al. Prevalence of methicillin-resistant Staphylococcus aureus as an etiology of community-acquired pneumonia. Clin Infect Dis. 2012;54(8):1126-33.

9. Schramm GE, Johnson JA, Doherty JA, Micek ST, Kollef MH. Methicillinresistant Staphylococcus aureus sterile-site infection: the importance of appropriate initial antimicrobial treatment. Crit Care Med. 2006;34(8):2069-74.

10. Kumar A, Ellis P, Arabi Y, Roberts D, Light B, Parrillo JE, et al. Initiation of inappropriate antimicrobial therapy results in a fivefold reduction of survival in human septic shock. Chest. 2009;136(5):1237-48.

11. Schreiber M, Chan C, Shorr A. Resistant pathogens in nonnosocomial pneumonia and respiratory failure: is it time to refine the definition of health-care-associated pneumonia? Chest. 2010;137:1283-8. 
12. Attridge RT, Frei CR, Restrepo MI, Lawson KA, Ryan L, Pugh MJ, et al. Guideline-concordant therapy and outcomes in healthcare-associated pneumonia. Eur Respir J. 2011;38(4):878-87.

13. Grenier C, Pepin J, Nault V, Howson J, Fournier X, Poirier MS, et al. Impact of guideline-consistent therapy on outcome of patients with healthcareassociated and community-acquired pneumonia. J Antimicrob Chemother. 2011;66(7):1617-24

14. Shorr AF, Myers DE, Huang DB, Nathanson BH, Emons MF, Kollef MH. A risk score for identifying methicillin-resistant Staphylococcus aureus in patients presenting to the hospital with pneumonia. BMC Infect Dis. 2013;13(1):268

15. Mortensen EM, Nakashima B, Cornell J, Copeland LA, Pugh MJ, Anzueto A, et al. Population-based study of statins, angiotensin II receptor blockers, and angiotensin-converting enzyme inhibitors on pneumonia-related outcomes. Clin Infect Dis. 2012;55(11):1466-73.

16. Mortensen EM, Halm EA, Pugh MJ, Copeland LA, Metersky M, Fine MJ, et al. Association of azithromycin with mortality and cardiovascular events among older patients hospitalized with pneumonia. JAMA. 2014;311(21):2199-208

17. Charlson ME, Pompei P, Ales KL, MacKenzie CR. A new method of classifying prognostic comorbidity in longitudinal studies: development and validation. J Chron Dis. 1987;40(5):373-83.

18. Deyo RA, Cherkin DC, Ciol MA. Adapting a clinical comorbidity index for use with ICD-9-CM administrative databases. J Clin Epidemiol. 1992:45(6):613-9.

19. Mandell LA, Wunderink RG, Anzueto A, Bartlett JG, Campbell GD, Dean NC, et al. Infectious Diseases Society of America/American Thoracic Society consensus guidelines on the management of community-acquired pneumonia in adults. Clin Infect Dis. 2007:44(Supplement 2):S27-72.

20. Mortensen EM, Coley CM, Singer DE, Marrie TJ, Obrosky DS, Kapoor WN, et al. Causes of death for patients with community-acquired pneumonia: results from the Pneumonia Patient Outcomes Research Team cohort study. Arch Intern Med. 2002;162(9):1059-64

21. Sohn MW, Arnold N, Maynard C, Hynes DM. Accuracy and completeness of mortality data in the Department of Veterans Affairs. Popul Health Metrics. 2006:4:2

22. Brito $V$, Niederman MS. Healthcare-associated pneumonia is a heterogeneous disease, and all patients do not need the same broadspectrum antibiotic therapy as complex nosocomial pneumonia. Curr Opin Infect Dis. 2009:22(3):316-25.

23. Ewig S, Welte T, Torres A. Is healthcare-associated pneumonia a distinct entity needing specific therapy? Curr Opin Infect Dis. 2012;25(2):166-75.

24. Shorr AF, Zilberberg MD, Reichley R, Kan J, Hoban A, Hoffman J, et al. Validation of a clinical score for assessing the risk of resistant pathogens in patients with pneumonia presenting to the emergency department. Clin Infect Dis. 2012;54(2):193-8.

25. Shorr AF, Zilberberg MD, Micek ST, Kollef MH. Prediction of infection due to antibiotic-resistant bacteria by select risk factors for health care-associated pneumonia. Arch Intern Med. 2008;168:2205-10.

26. Chalmers JD, Rother C, Salih W, Ewig S. Healthcare-associated pneumonia does not accurately identify potentially resistant pathogens: a systematic review and meta-analysis. Clin Infect Dis. 2014;58(3):330-9.

27. Madaras-Kelly KJ, Remington RE, Sloan KL, Fan VS. Guideline-based antibiotics and mortality in healthcare-associated pneumonia. J Gen Intern Med. 2012;27(7):845-52.

28. Aliberti S, Di Pasquale M, Zanaboni AM, Cosentini R, Brambilla AM, Seghezzi $S$, et al. Stratifying risk factors for multidrug-resistant pathogens in hospitalized patients coming from the community with pneumonia. Clin Infect Dis. 2012:54(4):470-8

29. Minejima E, Lou M, Nieberg P, Wong-Beringer A. Patients presenting to the hospital with MRSA pneumonia: differentiating characteristics and outcomes with empiric treatment. BMC Infect Dis. 2014;14(1):252

30. Jung WJ, Kang YA, Park MS, Park SC, Leem AY, Kim EY, et al. Prediction of methicillin-resistant Staphylococcus aureus in patients with non-nosocomial pneumonia. BMC Infect Dis. 2013;13(1):370

31. Liu C, Bayer A, Cosgrove SE, Daum RS, Fridkin SK, Gorwitz RJ, et al. Clinical practice guidelines by the Infectious Diseases Society of America for the treatment of methicillin-resistant Staphylococcus aureus infections in adults and children. Clin Infect Dis. 2011;52(3):e18-55.

32. Centers for Disease Control and Prevention. Severe methicillin-resistant Staphylococcus aureus community-acquired pneumonia associated with influenza-Louisiana and Georgia, December 2006-January 2007. MMWR Morb Mortal Wkly Rep. 2007;56(14):325-9.
33. Francis JS, Doherty MC, Lopatin U, Johnston CP, Sinha G, Ross T, et al. Severe community-onset pneumonia in healthy adults caused by methicillin-resistant Staphylococcus aureus carrying the Panton-Valentine leukocidin genes. Clin Infect Dis. 2005;40(1):100-7.

34. Hageman JC, Uyeki TM, Francis JS, Jernigan DB, Wheeler JG, Bridges CB, et al. Severe community-acquired pneumonia due to Staphylococcus aureus, 2003-04 influenza season. Emerg Infect Dis. 2006;12(6):894-9.

35. Kallen AJ, Brunkard J, Moore Z, Budge P, Arnold KE, Fosheim G, et al. Staphylococcus aureus community-acquired pneumonia during the 2006 to 2007 influenza season. Ann Emerg Med. 2009;53(3):358-65.

36. Lobo LJ, Reed KD, Wunderink RG. Expanded clinical presentation of community-acquired methicillin-resistant Staphylococcus aureus pneumonia. Chest. 2010;138(1):130-6.

37. Schweizer ML, Eber MR, Laxminarayan R, Furuno JP, Popovich KJ, Hota B, et al. Validity of ICD-9-CM coding for identifying incident methicillinresistant Staphylococcus aurues (MRSA) infections: is MRSA infection coded as a chronic disease? Infect Control Hosp Epidemiol. 2010;32(2):148-54

38. Schaefer MK, Ellingson K, Conover C, Genisca AE, Currie D, Esposito T, et al. Evaluation of International Classification of Diseases, Ninth Revision, Clinical Modification Codes for reporting methicillin-resistant Staphylococcus aureus infections at a hospital in Illinois. Infect Control Hosp Epidemiol. 2010;31(5):463-8.

39. Caffrey AR, Quilliam BJ, LaPlante KL. Comparative effectiveness of linezolid and vancomycin among a national cohort of patients infected with methicillin-resistant Staphylococcus aureus. Antimicrob Agents Chemother. 2010;54(10):4394-400.

40. Frei CR, Makos BR, Daniels KR, Oramasionwu CU. Emergence of communityacquired methicillin-resistant Staphylococcus aureus skin and soft tissue infections as a common cause of hospitalization in United States children. J Pediatr Surg. 2010;45:1967-74.

\section{Submit your next manuscript to BioMed Central and take full advantage of:}

- Convenient online submission

- Thorough peer review

- No space constraints or color figure charges

- Immediate publication on acceptance

- Inclusion in PubMed, CAS, Scopus and Google Scholar

- Research which is freely available for redistribution 